\title{
Heartbeat: Healthcare approaches to reducing adverse outcomes in patients with atrial fibrillation
}

doi:10.1136/heartjnl-2017-312538

Catherine M Otto

Atrial fibrillation (AF) affects over 30 million people worldwide. The increased risk of stroke and mortality associated with AF accounts for an increasing global burden of disease, both in terms of adverse outcomes and resultant costs. In standard medical practice, adherence to guideline based preventative anticoagulation is suboptimal. An integrated care model, using a multidisciplinary team and community support to provide patient-centred care, has been proposed to improve outcomes in the AF population. In a systematic review and meta-analysis that included $1383 \mathrm{AF}$ patients, Gallagher and colleagues ${ }^{1}$ found that an integrated care approach was associated with a reduction in both all-cause mortality (OR 0.51, 95\% CI 0.32 to $0.80, \mathrm{p}=0.003)$ and cardiovascular hospitalisations (OR 0.58, 95\% CI 0.44 to $0.77, p=0.0002$ ) (figure 1 ). However, there was no difference between integrated and standard care for endpoints of AF-related hospitalisations or cerebrovascular events.

In an editorial, Stewart ${ }^{2}$ stresses the contribution of AF to the epidemiology of cardiovascular disease given that the "population prevalence ranges from $2.3 \%-3.4 \%$ with a marked gradient in cases according to age. It is on this basis that $10 \%-20 \%$ of individuals aged $>65$ years develop AF with a lifetime risk of one in four." He also points out that despite the theoretical benefits of an integrated care approach for AF patients, there is little objective data to support its widespread application at this time (figure 2). He concludes: "To bring 'order' to this chaotic rhythm disorder via integrated management, therefore, there is still much to be done; particularly in respect to cost-effectively dealing with what is likely to be an increasingly older and more clinically complex patient population in whom the margin of error (in terms of benefits vs risk) is likely to become narrower.”

Correspondence to Professor Catherine M Otto, Division of Cardiology, University of Washington, Seattle, Washington, USA; cmotto@uw.edu

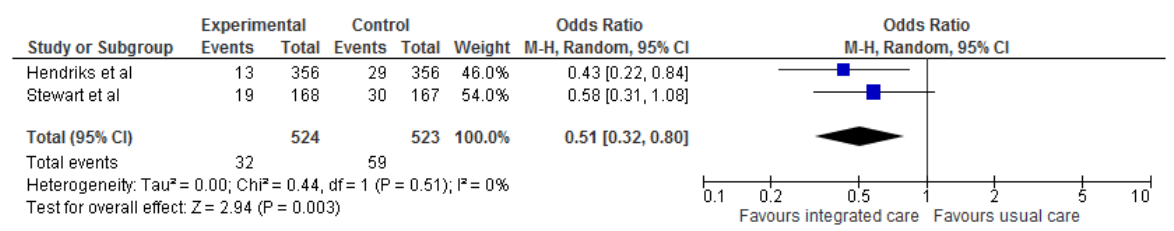

Figure 1 Impact of integrated care on all-cause mortality. M-H, Mantel-Haenszel method.
Also in this issue, Wong and colleagues ${ }^{3}$ examined data from almost 40000 women, with a mean age at baseline of 50 years, to study whether menopause is associated with an increased incidence of AF. Based on 1350 AF events over 20.5 years of follow-up, oestrogen-only hormone replacement therapy was associated with an increased risk of AF (HR 1.22; 95\% CI 1.02 to 1.45 ) but there were no significant differences based on age at menopause or use of combined hormone therapy.

In the accompanying editorial, Guhl and Magnani ${ }^{4}$ remind us that, despite this encouraging study in terms of AF risk, "Menopausal age is established as a strong marker of adverse cardiovascular risk. Earlier age of menopause has been related to increased myocardial infarction, stroke, heart failure and cardiovascular mortality" (figure 3).

The role of revascularisation versus medical therapy for older adults with non-ST-elevation acute coronary syndrome (NSTEACS) remains controversial. In a meta-analysis of four randomised trials and three observational studies that included over 20000 patients aged 75 years or older, Gnanenthiran and colleagues ${ }^{5}$ found that a routine invasive strategy reduced the risk of death (OR 0.67 , CI 0.61 to 0.74 ), myocardial infarction (OR 0.56, CI 0.45 to 0.70 ) and stroke (OR 0.53, CI 0.30 to 0.95 ) but resulted

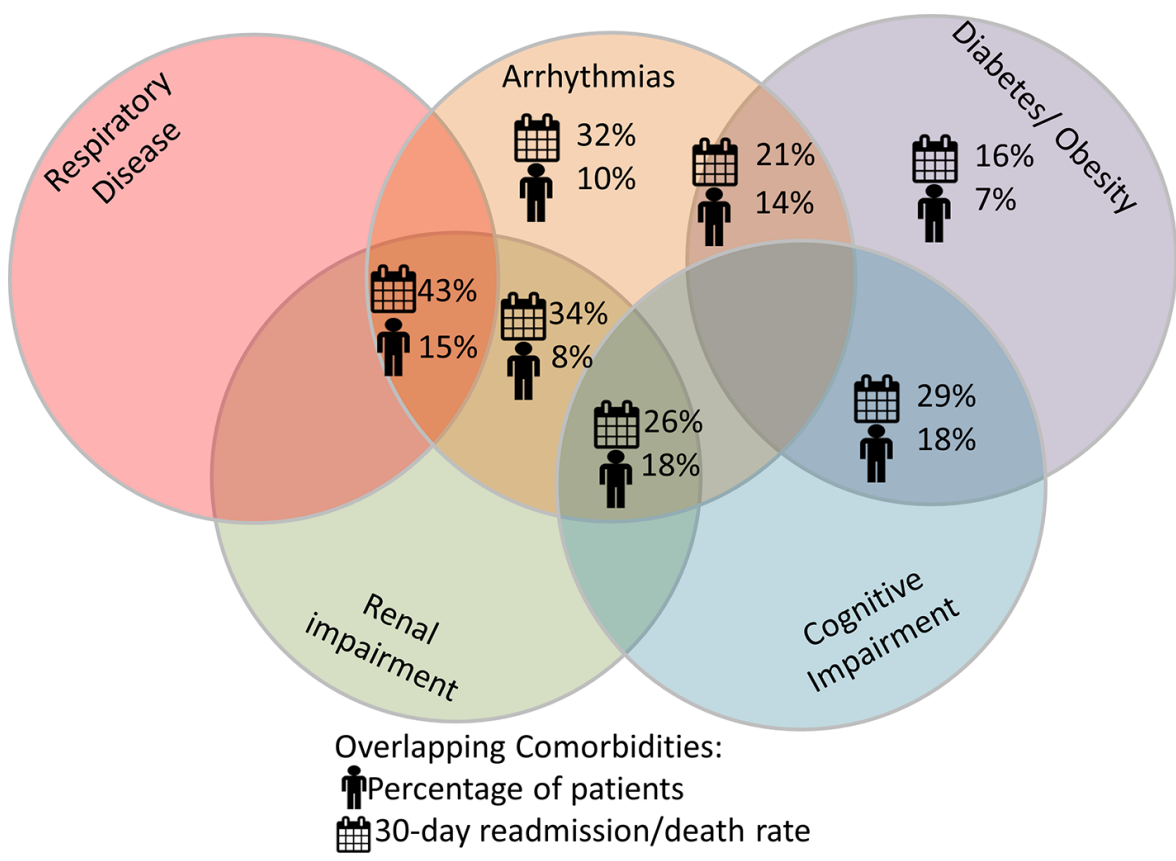

Figure 2 Impact of AF on 30-day outcomes when combined with other common comorbid conditions in patients hospitalised with HF. AF, atrial fibrillation; HF, heart failure. 


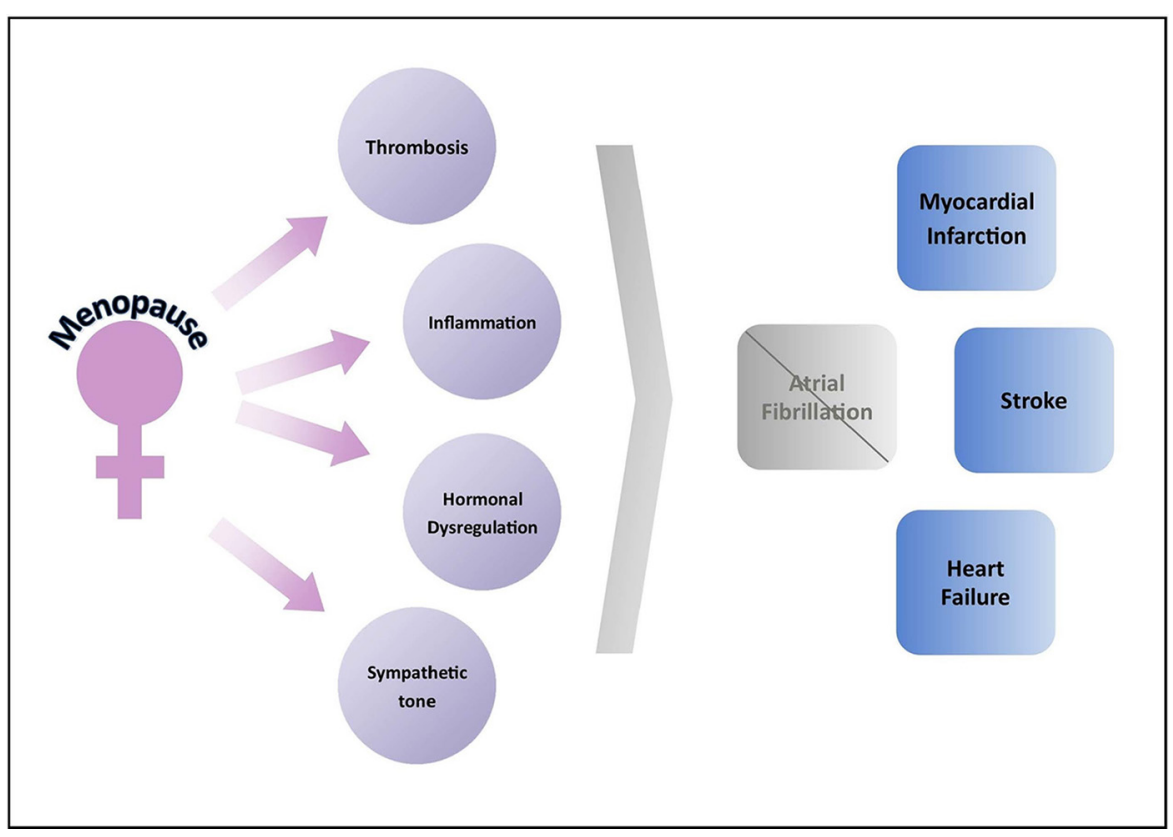

Figure 3 The figure presents a hypothesised association between age of menopause and cardiovascular outcomes, emphasising the consistent absence of a relation to atrial fibrillation as borne out by multiple epidemiological studies.

in a greater than twofold increased risk of major bleeding (figure 4).

De Carlo and Liga $^{6}$ comment that "While adequately-powered randomised define the most appropriate approach in elderly patients with NSTEACS, the meta-analysis by Gnanenthiran et $a l^{5}$ provides new, good-quality evidence supporting an early invasive strategy, which was associated with a significant reduction in most clinical hard endpoints. In this context, strategies able to limit the occurrence of bleeding complications (ie, trans-radial approach) and to better guide revascularisation (ie, invasive assessment of coronary flow reserve in the case of multivessel disease) should be implemented to maximise the clinical benefit and contain the risks of a routine invasive approach."

A set of two review articles in this issue discuss the vexing problem of prosthetic valve thrombosis, both with surgically implanted bioprosthetic and mechanical valves $^{7}$ and with transcatheter bioprosthetic valves $^{8}$ (figure 5). These summaries of the current literature can help guide future clinical research on this important complication of heart valve replacement.

The Education in Heart article ${ }^{9}$ in this issue discusses cardiac resynchronisation therapy for management of heart failure with reduced ejection fraction. The pathophysiologic rationale, patient selection, and procedural technique are summarised concisely in this article. The Image Challenge ${ }^{10}$ shows an apical cyst on echocardiography and chest CT in a

\section{A. In-hospital Mortality}

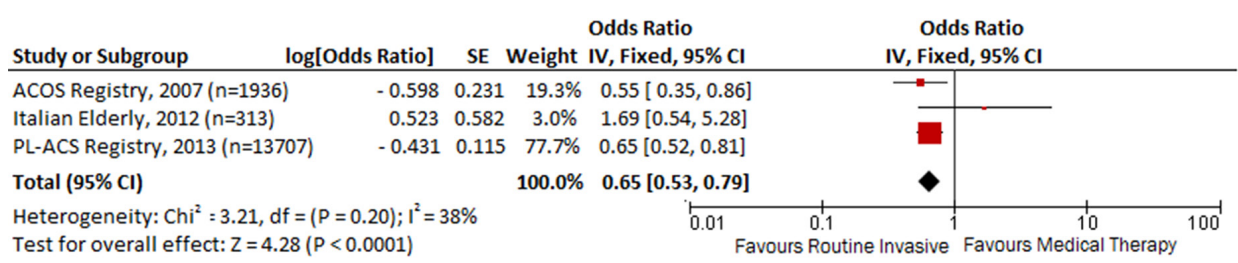

\section{B. Mortality at Follow-up}

Figure 4 Mortality after NSTEACS in adults aged 75 years and older, comparing a routine invasive approach with medical therapy. 


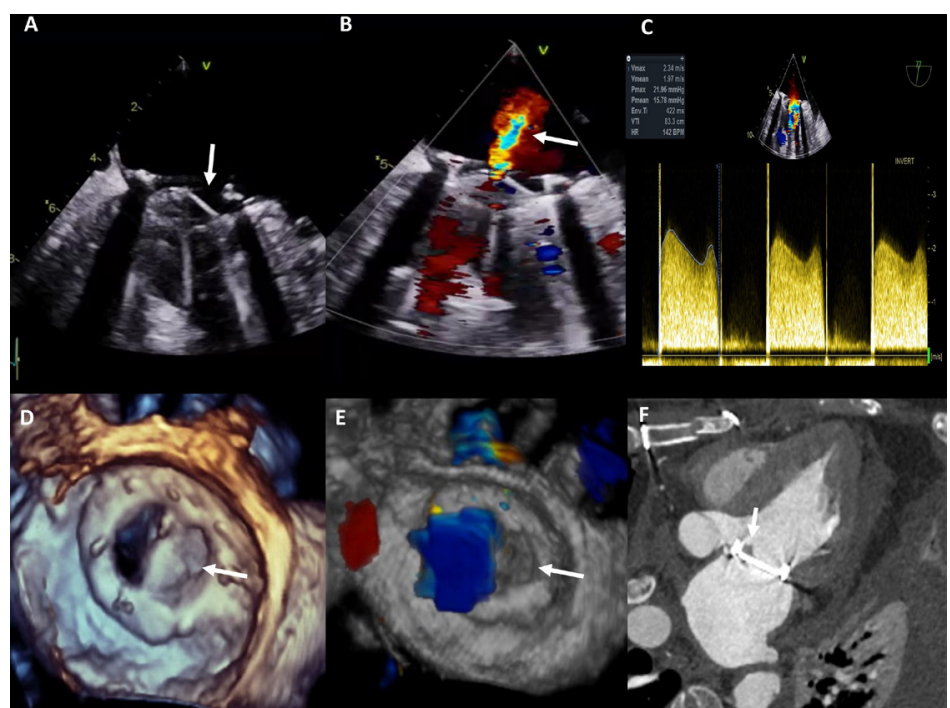

Figure 5 (A) Bileaflet mechanical mitral valve replacement. During diastole, only one leaflet opens. (B) Central jet of pathological regurgitation (arrow). (C) Increased transmitral gradient $16 \mathrm{~mm} \mathrm{Hg}$. (D) Three-dimensional view of mitral valve from atrial side. One orifice is fixed and occluded (arrow). (E) Absence of colour Doppler through occluded orifice. (F) CT demonstrating $1 \mathrm{~cm}$ thrombus partially occluding leaflet (arrow).

76-year-old woman with anaemia. See if you can make the diagnosis from these images!

Competing interests None declared.

Provenance and peer review Commissioned; internally peer reviewed.
(C) Article author(s) (or their employer(s) unless otherwise stated in the text of the article) 2017. All rights reserved. No commercial use is permitted unless otherwise expressly granted.

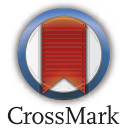

To cite Otto CM. Heart 2017:103:1925-1927.

Heart 2017:103:1925-1927

doi:10.1136/heartjnl-2017-312538

\section{REFERENCES}

1 Gallagher C, Elliott AD, Wong CX, et al. Integrated care in atrial fibrillation: a systematic review and metaanalysis. Heart 2017;103:1947-53.

2 Stewart S. Bringing order to chaos: the case for integrated management of atrial fibrillation. Heart 2017;103:1928-9.

3 Wong JA, Rexrode KM, Sandhu RK, et al. Menopausal age, postmenopausal hormone therapy and incident atrial fibrillation. Heart 2017;103:1954-61.

4 Guhl EN, Magnani JW. Atrial fibrillation and menopause: something else to worry about, or not? Heart 2017;103:1930-1.

5 Gnanenthiran SR, Kritharides L, D'Souza M, et al. Revascularisation compared with initial medical therapy for non-ST-elevation acute coronary syndromes in the elderly: a meta-analysis. Heart 2017; 103:1962-9.

6 De Carlo M, Liga R. Elderly patients with non-STelevation acute coronary syndromes: a call for action. Heart 2017;103:1932-3.

7 Lim WY, Lloyd G, Bhattacharyya S. Mechanical and surgical bioprosthetic valve thrombosis. Heart 2017; 103:1934-41.

8 Nakatani S. Subclinical leaflet thrombosis after transcatheter aortic valve implantation. Heart 2017; 103:1942-6.

9 Rao P, Faddis M. Cardiac resynchronisation therapy: current indications, management and basic troubleshooting. Heart 2017;103:2000-7.

10 Oh JK, Park JH. A 76-year-old woman with anaemia and cardiomegaly. Heart 2017;103:1999. 